\title{
Radio and Optical Observations at High Spatial and Spectral Resolution of Compact Young Planetary Nebulae
}

\author{
L.F. Miranda ${ }^{1}$, J.M. Torrelles ${ }^{2}$ and C. Eiroa $^{3}$ \\ ${ }^{1}$ Universidad Complutense de Madrid; ${ }^{2}$ Instituto de Astrofísica de Andalucía, CSIC; \\ ${ }^{3}$ Universidad Autónoma de Madrid
}

We present VLA-A radio continuum at $3.6 \mathrm{~cm}$ and $\mathrm{H} 92 \alpha$ observations, and optical longslit spectra at high spectral and spatial resolution of the compact Planetary Nebulae (PNs) Hu 2-1, IC 4997, IC 5117 and NGC 6884. The radio continuum maps show the subarcsecond structure of the PNs in detail. From the optical spectra, the basic kinematical properties can be deduced. The H92 $\alpha$ emission line, spatially and spectrally resolved in IC 5117 and Hu 2-1, allows us to observe the kinematics of the PNs in spatial scales of $\sim 0.3^{\prime \prime}$. By combining the radio and optical data, it is possible to identify the components present in the nebulae, to deduce their spatio-kinematical structure, and to obtain estimates for the electron density and temperature, ionized masses and kinematical ages. All this information permits to impose constraints about the mass ejection processes involved in the formation of PNs and when they have occurred.

Some results obtained for the observed PNs are the following. Hu 2-1 is a bipolar PN with three highly collimated condensations. A geometrically thick toroid, probably charaterized by $\mathrm{N}$ overabundance, is observed in the equatorial plane of Hu 2-1 (Miranda 1995). IC 4997 is an extremely young double-shell PN consisting of an hour-glass outer shell formed $\sim 670$ years ago, and a circular inner shell ejected $\sim 65$ years ago (Miranda, Torrelles \& Eiroa 1996). IC 5117 is a very compact (size $\left.\sim 1^{\prime \prime}\right)$ young $(\sim 350$ years) cylindrical PN. The H92 $\alpha$ emission line and radio continuum emission suggest variations of the electron density in scales of $\sim 0.4^{\prime \prime}$ (Miranda, Torrelles \& Eiroa 1995). NGC 6884 presents an extremely knotty morphlogy at $3.6 \mathrm{~cm}$. The optical spectra indicate that the shell of NGC 6884 does not present spherical symmetry. In addition, the [NII] emission is noticeably enhanced in two regions which are located diametrically opposited to the central star and cannot be described with a simple (e.g. toroidal or ring-like) geometry (Miranda et al. 1996, in preparation).

\section{REFERENCES}

Miranda, L.F., 1995, A\&A, 304, 531

Miranda, L.F., Torrelles, J.M., Eiroa, C., 1995, ApJ, 446, L39

Miranda, L.F., Torrelles, J.M., Eiroa, C., 1996 ApJ, 461, L111 